Background Patients with severe Inflammatory Bowel Disease (IBD), are prescribed anti-TNF- $\alpha$ agents, if clinical need necessitates, whose immunosuppressive action can potentially reactivate latent tuberculosis infections (LTBI). Meticulous pre antiTNF- $\alpha$ LTBI screening and management in accordance with the British Thoracic Society's (BTS) guidelines is imperative for patient safety and public health.

Objective A retrospective clinical audit was performed to evaluate the performance of University Hospital Southampton's gastrointestinal department in screening for LTBI in patients with IBD. The performance of LTBI screening was compared to the BTS standards.

Method The audit population was obtained using the gastrointestinal department's biologics database. Inclusion criteria included patients who started their first anti-TNF- $\alpha$ agent between 01/01/2006 to 04/11/2016. Exclusion criteria included deceased patients and patients screened by alternative departments/trusts. Extent of LTBI screening was assessed using hospital record systems: EDocs, EQuest, ECamis and Spectra PACS. If evidence of screening was not located, then this was considered as a failure to meet standard. Following statistical analysis, comparisons were made with BTS standards.

Results Of the 471 patients audited, $51.2 \%$ were females and 48.8\% males. $75.2 \%$ CD patients and $24.8 \%$ UC patients. 231 patients' (49\%) LTBI screening was insufficient. 157 patients (33.3\%) lacked an adequate TB history and 94 patients (20\%) failed to have a chest radiograph (CXR) within 3 months of therapy commencement. Additionally, 85 patients $(18.3 \%)$ failed to have an IGRA performed. 15 patients (3.2\%) were diagnosed with LTBI, whilst 1 case of TB reactivation, occurred once immunosuppressive therapy had commenced.

Conclusion The completeness of LTBI screening in the audited group was suboptimal with deficits in TB history performance, CXR, TST and IGRAs. One case of active miliary TB occurred as a result of inadequate screening. In light of this, recommendations to address deficits and ultimately improve screening were proposed.

\section{P164 QFT-PLUS: DO THE PEPTIDES IN THE TB2 TUBE INDUCE A T SUPPRESSOR RESPONSE IN SOME SUBJECTS?}

${ }^{1} \mathrm{M}$ Sieren, ${ }^{1} \mathrm{~B}$ Thippeswamy, ${ }^{2} \mathrm{C}$ Mandiveyi, ${ }^{2} \mathrm{~N}$ Marshall, ${ }^{1} \mathrm{G}$ Bothamley. ${ }^{1}$ Department of Respiratory Medicine, Homerton University Hospital, London, UK; ${ }^{2}$ Microbiology Department, Homerton University Hospital, London, UK

\subsection{6/thoraxjnl-2017-210983.306}

Introduction The revised QuantiFERON-TB Gold assay (QFTPlus) has an extra tube (TB2), containing peptides thought to stimulate CD8 + T cells; TB7 antigen is no longer included. The test is designed to detect latent tuberculosis infection.

Hypothesis Levels of interferon- $\gamma$ in TB2 should always be greater than TB1

Methods QFT-Plus was used routinely in a TB contact clinic June 2016 - January 2017. Interferon- $\gamma$ values were recorded minus the negative control; a significant difference was defined as a two-fold variation. Clinical details were recorded after consent on a standard form, which included age, sex, reason for test, tuberculin response $(\mathrm{mm})$ and HIV status.

Results Data was available from 259 subjects; 2 were HIV-positive. Four gave an indeterminate result. The majority (194; $75 \%$ ) gave a negative test for both tubes. For 65 positive tests with $\mathrm{a} \geq 2$ fold difference (all $\geq 0.7 \mathrm{IU} / \mathrm{mL}$ difference),
TB1 $\geq$ TB2 occurred in $4(6 \%)$ and $\mathrm{TB} 2 \geq \mathrm{TB} 1$ occurred in 3 (5\%). All 4 TB1 $\geq \mathrm{TB} 2$ had no TB2 response: there were 2 contacts (10 and $0.68 \mathrm{IU} / \mathrm{ml})$; one was PPD positive with neck lymphadenopathy $(4.23 \mathrm{IU} / \mathrm{ml})$; one was PPD negative with haemoptysis after nasal surgery $(1.75 \mathrm{IU} / \mathrm{ml})$. Where TB2 was positive in the 'grey zone' and TB1 negative, one had Crohn's disease $(0.24,0.01 \mathrm{IU} / \mathrm{ml})$ and the other $(0.23,0.01$ $\mathrm{IU} / \mathrm{ml}$ ) was a contact of non-pulmonary $\mathrm{TB}$, but in none was there HIV co-infection. Three had TB2 $\geq$ TB1: one with smear-positive lung TB had a TB2 $=0.6$ with a TB1 $=0.25$, but the latter was in an accepted 'grey zone'; two had positive tests for both tubes, of which one was a contact 30 years previously and one had testicular TB.

Conclusion These data may indicate a technical failure of the TB2 tube. Alternatively, the absent immune response, associated with active disease, might prove useful in determining who could benefit from preventive treatment.

\begin{tabular}{llll}
\multicolumn{5}{l}{ Abstract P164 Table 1} \\
\hline \multicolumn{4}{l}{ Highest TB1 or TB2 value $($ IU $/ \mathrm{ml})$} \\
\hline Difference (IU/ml) & $<0.2(\mathrm{n}=186)$ & $\geq 0.2$ and $<0.35(\mathrm{n}=4)$ & $>0.35(\mathrm{n}=65)$ \\
\hline $0-0.1$ & 182 & 2 & 31 \\
$>0.1$ to $<0.35$ & 4 & 2 & 12 \\
$\geq 0.35$ & 0 & 0 & 22 \\
$>2$-fold & na & 2 & 7 \\
\hline
\end{tabular}

\section{P165 LATENT TUBERCULOSIS INFECTIONS (LTBI) NATIONAL SCREENING PROGRAMME}

${ }^{1}$ YO Abunga, 'M Day, ${ }^{2} \mathrm{~N}$ Wright, ${ }^{1} \mathrm{SO}$ Brij. 'Peterborough City Hospital, Peterborough, UK; ${ }^{2}$ Peterborough CCG, Peterborough, UK

\subsection{6/thoraxjnl-2017-210983.307}

Background The new entrant TB screening programme identified very few persons for Latent TB screening locally. However, following national funding for community LTBI screening, 12 GP practices were identified to undertake IGRA testing in "high risk" registered persons.

Aims To evaluate the feasibility and clinical outcomes of the LTBI National Screening Programme

Methods From May 2016 until April 2017, persons from countries with high TB incidence $(\geq 150 / 100,000$ population) and sub-Saharan Africa were identified by the GP practices and offered LTBI screening with IGRA if they had arrived in the UK in the last 5 years and were aged 16-35 years. IGRA Results were forwarded to the TB team at the local hospital. Persons with positive, borderline positive and indeterminate Results were offered appointments for assessment and treatment in the TB Clinic.

Results 360 persons were community-screened for LTBI. Of the 360 persons, 305 (84.7\%) had non-reactive IGRA Results and therefore did not require follow up. The remaining 55 $(15.3 \%)$ had either positive, borderline positive or 2 indeterminate Results and were referred to the local TB team. Of 55 persons referred 2 did not attend any appointments offered and 53 attended 2 of whom were subsequently found to have active TB. The treatment completion rate was $89.2 \%$ (see Table 1). Despite the inclusion criteria, of the 55 persons offered consultation, 16 had been residing in the UK for more than 5 years and 7 were over the age of 35 . 
Conclusion Retrospective case-finding in General Pracice is feasible and the uptake for screening appeared very successful. Inclusion criteria were not strictly adhered to but identified persons with LTBI were offered non-funded treatment. Treatment completion rates were excellent. These Results will hopefully inspire on-going prospective screening of newly registered persons and prove to be more successful in the longterm than new entrant screening.

\begin{tabular}{|c|c|c|}
\hline IGRA results & Outcomes & $\begin{array}{l}\text { Number of } \\
\text { persons }\end{array}$ \\
\hline $\begin{array}{l}\text { Negative and borderline } \\
\text { negative }\end{array}$ & Discharged & 298 \\
\hline $\begin{array}{l}\text { Technical error/ } \\
\text { insufficient cells }\end{array}$ & Discharged & 7 \\
\hline Indeterminate & Referred to the local TB clinic & 5 \\
\hline \multirow{12}{*}{$\begin{array}{l}\text { Positive and borderline } \\
\text { positive }\end{array}$} & Referred to the local TB clinic & 50 \\
\hline & $\begin{array}{l}\text { Outcomes of the } 55 \text { referred to the } \\
\text { local TB clinic }\end{array}$ & \\
\hline & Diagnosis of active TB & 2 \\
\hline & Completed LTBI treatment & 33 \\
\hline & Stopped LTBI treatment & 2 \\
\hline & Declined LTBI treatment & 2 \\
\hline & Postponed treatment - pregnancy & 2 \\
\hline & Postponed treatment - other & 2 \\
\hline & $\begin{array}{l}\text { Not suitable for treatment } \\
\text { (previous active/latent treatment or due to } \\
\text { age) }\end{array}$ & 7 \\
\hline & Negative IGRA - 2nd test & 1 \\
\hline & DNA & 2 \\
\hline & Lost to follow up & 2 \\
\hline
\end{tabular}

\section{P166 DIAGNOSING PULMONARY TUBERCULOSIS: HOW USEFUL IS THE CHEST X-RAY REPORT?}

${ }^{1} \mathrm{KJ}$ Myall, ${ }^{2} \mathrm{~W}$ Owen, ${ }^{2} \mathrm{RA}$ Breen, ${ }^{1} \mathrm{~F}$ Perrin. ' $K$ King's College Hospital, London, UK; ${ }^{2}$ Guy's and St Thomas' NHS Foundation Trust, London, UK

\subsection{6/thoraxjnl-2017-210983.308}

Introduction and Objectives The diagnosis of pulmonary tuberculosis (TB) is frequently delayed, resulting in increased morbidity and mortality. One factor may be a delay in referral for chest X-ray (CXR). The NICE quality statement of January $2017^{1}$ suggests that all patients with imaging suggestive of active pulmonary tuberculosis are assessed by the next working day. We wanted to understand how many patients with confirmed TB have an initial CXR which correctly reported the diagnosis.

Methods We performed a retrospective analysis of the patient databases of two London hospitals of patients with confirmed pulmonary TB. We examined the report of the CXR prior to diagnosis in each case for mention of tuberculosis or referral to respiratory medicine.

Results 247 adult patients were treated for pulmonary TB between January 2014 and May 2017. Of these, 231 (94\%) had a CXR reported at our hospitals prior to diagnosis. 206 (89\%) patients had a CXR which was reported as abnormal and 106 (52\%) had a report which suggested TB as the diagnosis. In $29(12 \%)$, the report did not mention TB, but did suggest referral to a respiratory physician, meaning that overall, $65 \%$ of cases would have been assessed. 198 patients (86\%) had culture-positive disease, and of these, 91 (46\%) had a CXR which suggested the diagnosis. In 123 cases (50\%), the sputum was smear-positive for AFB and of these, 63 (51\%) had a CXR reported as TB. Of those patients whose CXR was reported as TB, $92(87 \%)$ had a positive culture, and 63 (51\%) were smear-positive.

Conclusions In our study, the initial CXR was abnormal in most cases of pulmonary tuberculosis, but in only $52 \%$ of these was TB suggested as the diagnosis. Thus almost half of diagnoses were missed on initial CXR, including those with smear-positive disease, and an automatic referral for assessment would not have been triggered. We think that these data highlight the importance of considering radiologist training as part of TB control efforts.

\section{REFERENCE}

1 National Institute for Health and Care Excellence. Tuberculosis 2017;QS141. Available at: nice.org.uk/guidance/qs141

\section{P167 THE USEFULNESS OF TABLET COUNTING TO IDENTIFY POTENTIAL TB TREATMENT NON-COMPLIANCE}

H Patel, Y Abunga, SO Brij. Peterborough City Hospital, Peterborough, UK

\subsection{6/thoraxjnl-2017-210983.309}

Background Early identification of non-compliance in patients taking anti-tuberculous therapy (ATT) may improve treatment outcomes and prevent the emergence of drug therapy resistance. Since 2012, the TB Pharmacy Team has been tablet counting to identify missed doses. Strategies to improve ATT compliance such as provision of dosette boxes, more frequent appointments and directly observed therapy (DOT) can then be initiated.

Aim To evaluate the usefulness of tablet counting as an effective strategy to help identify and reduce non-compliance with ATT.

Methods A retrospective review of active TB cases diagnosed between January 2012 to December 2016 was undertaken. Objective compliance was graded according to accuracy of tablet counting. Treatment outcomes were assessed clinically and subsequent change from normal practice identified.

Results 248 persons received at least 2 months ATT from the TB Clinic and were further evaluated. The majority of persons $(87.5 \%)$ were graded as fully $(61.3 \%)$ compliant (accurate tablet counting) or mostly (26.2\%) compliant (few missed doses) both with good clinical response. The commonest strategy to improve treatment outcome in $25(10.1 \%)$ with partial compliance (missed doses with adequate clinical response) was to increase duration of therapy. Poor compliance (missed doses, poor clinical response, more than 1 non-attendance) in 4 patients resulted in admission and completion of DOT as an in-patient. NICE guidance would have identified 22 persons for DOT. 19/22 received DOT (2 MDR-TB; 12 prisoners; 3 homelessness, 2 intravenous iv drug users). 3 (2 with mental health disorders; 1 alcohol dependence) successfully completed therapy with increased frequency of appointments and family involvement. Tablet counting identified 25 partially compliant persons (12 had no obvious risk factor; 5 prisoners on DOT; 2 persons with mental health disorders and 1 with alcohol dependence as above; 3 HIV; 2 pregnant). Treatment 